\title{
The Application of Ferric Chloride-Lignin Sulfonate as Shale Inhibitor in Water-Based Drilling Fluid
}

\author{
Rongjun Zhang ${ }^{1}$, Long Gao ${ }^{1}$, Wenguang Duan ${ }^{2}$, Weimin $\mathrm{Hu}^{1}$, Weichao Du ${ }^{1} \oplus$, Xuefan Gu ${ }^{1}$, \\ Jie Zhang ${ }^{1, *}$ and Gang Chen ${ }^{1,3, *}$ \\ 1 Shaanxi Province Key Laboratory of Environmental Pollution Control and Reservoir Protection Technology \\ of Oilfields, Xi'an Shiyou University, Xi'an 710065, China; rongjunzhang@xsyu.edu.cn (R.Z.); \\ gaolong22330@163.com (L.G.); cg1014@126.com (W.H.); duweichao@xsyu.edu.cn (W.D.); \\ xuefangu@xsyu.edu.cn (X.G.) \\ 2 CNPC Xibu Drilling Engineering Company Limited, Urumqi, Xinjiang 830026, China; \\ duanwg_zj20@cnpc.com.cn \\ 3 State Key Laboratory of Petroleum Pollution Control, CNPC Research Institute of Safety \\ and Environmental Technology, Beijing 102206, China \\ * Correspondence: zhangjie@xsyu.edu.cn (J.Z.); gangchen@xsyu.edu.cn (G.C.); Tel.: +86-029-88382690 (R.Z.)
}

Academic Editors: Claudia Crestini and Heiko Lange

check for

Received: 8 October 2019; Accepted: 19 November 2019; Published: 27 November 2019

\begin{abstract}
A series of ferric chloride-lignin sulfonate (FCLS) was prepared from ferric chloride and lignin sulfonate to be used as shale inhibitor. The swelling rate of clay with FCLS-2 $(w / w=0.3 \%)$ decreased to $41.9 \%$. Compared with control, FCLS-2 displayed high inhibitive ability against the hydrating and swelling processes of clay. Thus, the swelling degree of samples with FCLS-2 was much lower than that of the control, as well as the mud ball was more stable in FCLS-2 solution. Essentially, these excellent performances in inhibitor were assigned to the hydrogen bonding, electrostatic interaction and anchoring between FCLS-2 and other components. In addition, FCLS-2 has good compatibility with other common drilling fluid additives, and it can reduce the viscosity of systems, regardless of the room temperature or high temperature.
\end{abstract}

Keywords: ferric chloride; lignin sulfonate; inhibitor; shale; swelling

\section{Introduction}

Shale oil/gas has been one of the topic of interests in the world in recent years. Drilling is the first and most important process in the shale oil/gas exploration. Water-based drilling fluids are considered economical and environmentally friendly compared to synthetic and oil-based drilling fluids in the shale oil/gas exploration. Quite different from the normal reservoir, the stability of borehole suffers some problems mostly because of hydrating and swelling of water sensitive shale during the drilling processes, including borehole wash-out, stuck pipe, disintegration of cuttings, and bit balling [1-3]. Theoretically, when the water sensitive shale (high montmorillonite content) is immersed in water-based drilling fluid, the swelling or dispersing behavior of shale will occur rapidly, which relates to the chemical composition of shale and the drilling fluid. Thus, various shale inhibitors have been utilized widely in drilling operations, including polymeric, amine-based, ionic liquids, and surfactant-based shale inhibitors [4,5]. However, most traditional additives face some challenge because of their deficiency in the kindly environmental requirements [6], and the processing of waste after drilling is relative difficult and high cost, especially for the oil-based shale inhibitors [7]. Recently, natural organic chemicals have drawn much attention as shale inhibitor in crude oil extraction due to their advantages, such as friendly environmental instinct, high inhibition ability, stable rheological performance, and high lubrication [8-10]. Currently, natural products have been developed as shale 
inhibitor in many water-based oilfield working fluids owing to their superior compatibility. In this work, the inhibitive performance as well as probable mechanism of ferric chloride-lignin sulfonate agent were investigated by using mud balls immersing test, linear expansion experiments, particle distribution measurements, thermogravimetric analysis (TGA), and scanning electron microscopy image (SEM).

\section{Results and Discussion}

\subsection{Optimization of Reaction Conditions}

The effect of the mass ratio of ferric chloride and lignin sulfonate on the inhibitive performance was evaluated via the clay-swelling rate as shown in Table 1. Among the six formula, the minimal swelling rate of clay was obtained when the mass ratio of ferric chloride and lignin sulfonate was 4:1. Thus, FCLS-2 agent was selected in the following experiments.

Table 1. The conditions of synthesis ferric chloride-lignin sulfonate inhibitors.

\begin{tabular}{ccc}
\hline Name & Mass Ratio & Swelling Rate (\%) (60 Min) \\
\hline LS & $/$ & 52 \\
FC & $/$ & 65 \\
FCLS-1 & $2: 1$ & 54 \\
FCLS-2 & $4: 1$ & 42 \\
FCLS-3 & $6: 1$ & 44 \\
FCLS-4 & $8: 1$ & 44 \\
\hline
\end{tabular}

\subsection{Test of Swelling Inhibition}

The dynamic inhibiting performance of aforementioned FCLS against the swelling process of bentonite was measured via the linear swelling rate. As shown in Figure 1, the swelling rate of control increased dramatically during the first $10 \mathrm{~min}$, and then increased slightly during the further $50 \mathrm{~min}$. While, the swelling rates of systems with inhibitors decreased significantly compared with control and reached to a minimum in $0.3 \%$ FCLS-2 samples. It was noticed that the swelling rate does not agree with a linear relation with different concentration of FCLS-2. This phenomenon revealed the complex inhibition mechanism ascribed to the adsorption of ferric chloride-lignin sulfonate on the clay surface through co-interaction, including electrostatic interaction, hydrophobic interaction and hydrogen bonds based on an anchoring effect. Under this co-interaction, swelling rate of samples with FCLS-2 $(w / w=0.3 \%)$ dropped from $69.0 \%$ to $41.9 \%$.

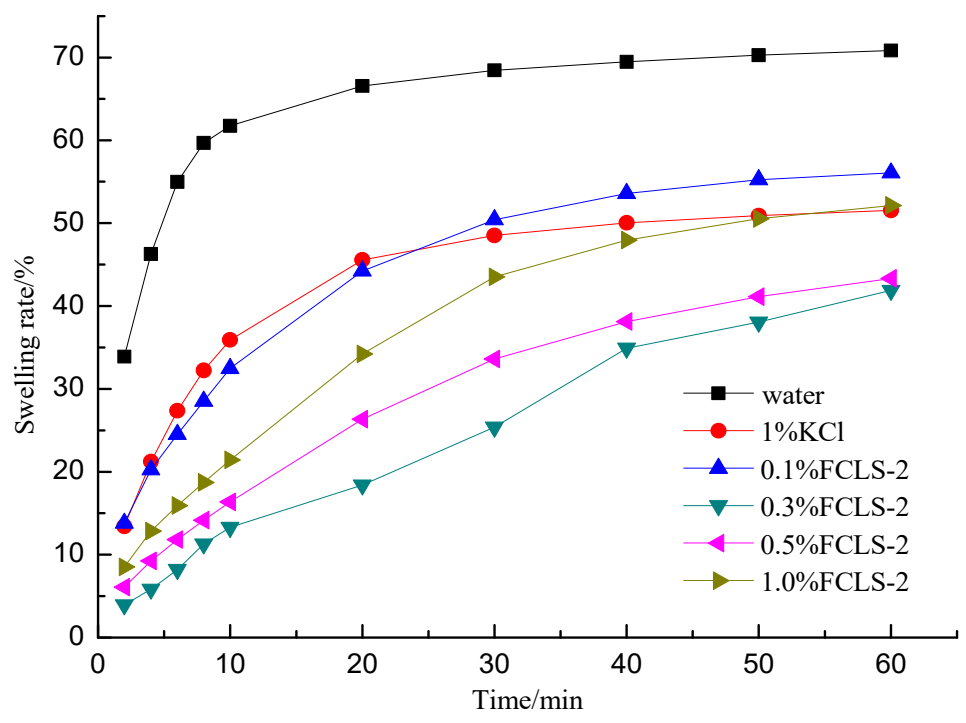

Figure 1. The effect of the inhibitors concentration. 
In addition, the mud ball test was employed to visually observe the effect of FCLS-2 on clay. As shown in Figure 2, the mud ball immersed in tap water evidently swelled within $12 \mathrm{~h}$, and its surface became completely rough and loose after $24 \mathrm{~h}$. While, the mud ball immersed in $0.3 \%$ FCLS-2 solution changed slightly in 12 h, 24 h, or 36 h, and its surface was still smooth except for a little crack after $36 \mathrm{~h}$ (Figure 3). The remarkable inhibition of FCLS-2 on the swelling process of clay mostly attribute to the absorption of FCLS-2 on the surface of clay, which blocked or reduced the water penetration into the clay, and prevent the clay from hydrating swelling.
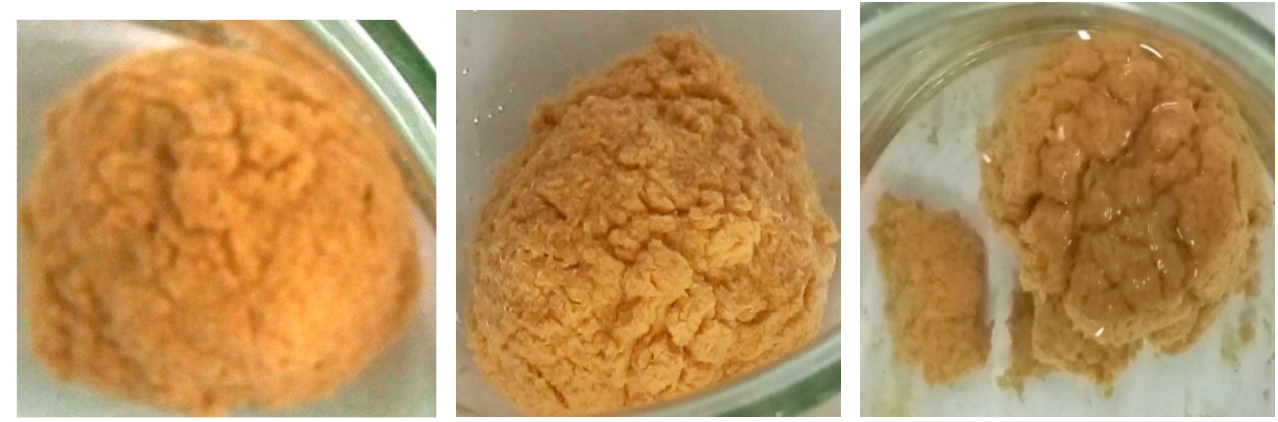

Figure 2. The appearance of mud balls immersed in water for $12 \mathrm{~h}, 24 \mathrm{~h}$, and $36 \mathrm{~h}$.
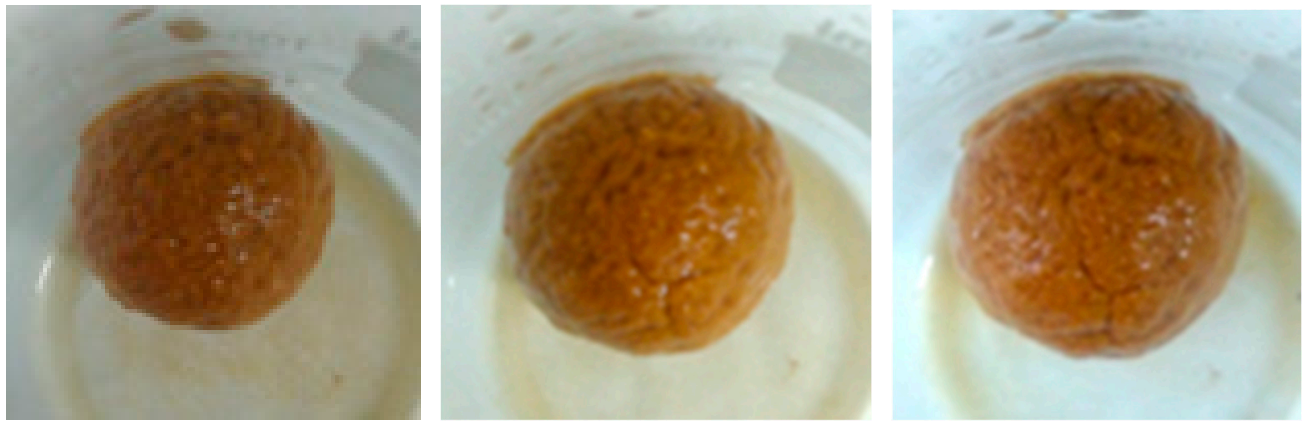

Figure 3. The appearance of mud balls immersed in FCLS-2 for $12 \mathrm{~h}, 24 \mathrm{~h}$, and $36 \mathrm{~h}$.

\subsection{Performance in Water-Based Drilling Fluids}

The performance of FCLS-2 in water-based drilling fluids was measured in accordance with GB/T 16783.1-2006, and the results are shown in Table 2.

Table 2. Evaluation results of drilling fluid rheological properties.

\begin{tabular}{|c|c|c|c|c|c|c|c|}
\hline \multirow{2}{*}{ Additives } & \multirow{2}{*}{$\mathrm{T} /{ }^{\circ} \mathrm{C}$} & AV & PV & $\mathbf{Y P}$ & $\mathrm{YP} / \mathrm{PV}$ & FL & \multirow{2}{*}{$\operatorname{tg}$} \\
\hline & & $/(\mathbf{m P a} \cdot \mathbf{s})$ & $/(\mathbf{m P a} \cdot \mathbf{s})$ & $/ \mathbf{P a}$ & $\mathrm{Pa} /(\mathrm{mPa} \cdot \mathrm{s})$ & $/ \mathrm{mL}$ & \\
\hline \multirow[b]{2}{*}{ Blank } & 25 & 3.6 & 2.1 & 1.40 & 0.67 & 12.0 & 0.07 \\
\hline & 120 & 2.6 & 1.8 & 0.70 & 0.39 & 14.0 & 0.18 \\
\hline \multirow{2}{*}{ PAM } & 25 & 8.0 & 4.2 & 3.88 & 0.92 & 13.0 & 0.03 \\
\hline & 120 & 7.8 & 4.8 & 3.30 & 0.74 & 15.7 & 0.03 \\
\hline PAM & 25 & 3.6 & 3.1 & 0.51 & 0.16 & 16.2 & 0.03 \\
\hline$+0.3 \%$ FCLS-3 & 120 & 6.0 & 4.5 & 1.20 & 0.25 & 15.2 & 0.04 \\
\hline \multirow{2}{*}{ Modified starch } & 25 & 4.9 & 2.8 & 2.15 & 0.77 & 11.0 & 0.09 \\
\hline & 120 & 9.8 & 8.5 & 2.50 & 0.15 & 15.7 & 0.11 \\
\hline \multirow{2}{*}{$\begin{array}{l}\text { Modified starch } \\
+0.3 \% \text { FCLS-3 }\end{array}$} & 25 & 3.5 & 2.2 & 1.33 & 0.60 & 10.1 & 0.19 \\
\hline & 120 & 5.7 & 4.5 & 1.20 & 0.55 & 15.5 & 0.11 \\
\hline
\end{tabular}

With nearly the same low fluid loss performance, the viscosity and shearing force of the drilling fluid added FCLS-2 became lower than the control sample without inhibitor, but the filtration property 
does not present any significant change, indicating that FCLS-2 can restrain the bentonite slurry deep swelling to reduce the viscosity effectively. The results also indicated that FCLS- 2 is compatible with the common drilling fluid additives, modified starch, and PAM, and it does not enhance the filtration as added in the composed water-based drilling fluid. The most important thing is that FCLS-2 can reduce the viscosity under high temperature, which can be used as a viscosity reducer.

\subsection{Particle Size Distribution Test}

The effect of FCLS-2 on bentonite particle size distribution was also studied since size distribution are closely related to reveal the swelling process. Figure 4 shows that the average size of original bentonite particles was $38 \mu \mathrm{m}$, and the hydrated samples reduced to $8 \mu \mathrm{m}$ (Table 3). When the LS or FCLS-2 was added in the hydrated bentonite respectively $16 \mathrm{~h}$ later, the average size of both the systems increased to some extent compared with the water-treated samples, especially the particle size of FCLS-2 treated samples. The obvious larger particle size in samples with FCLS- 2 was the consequence of the flocculation function of FCLS-2.

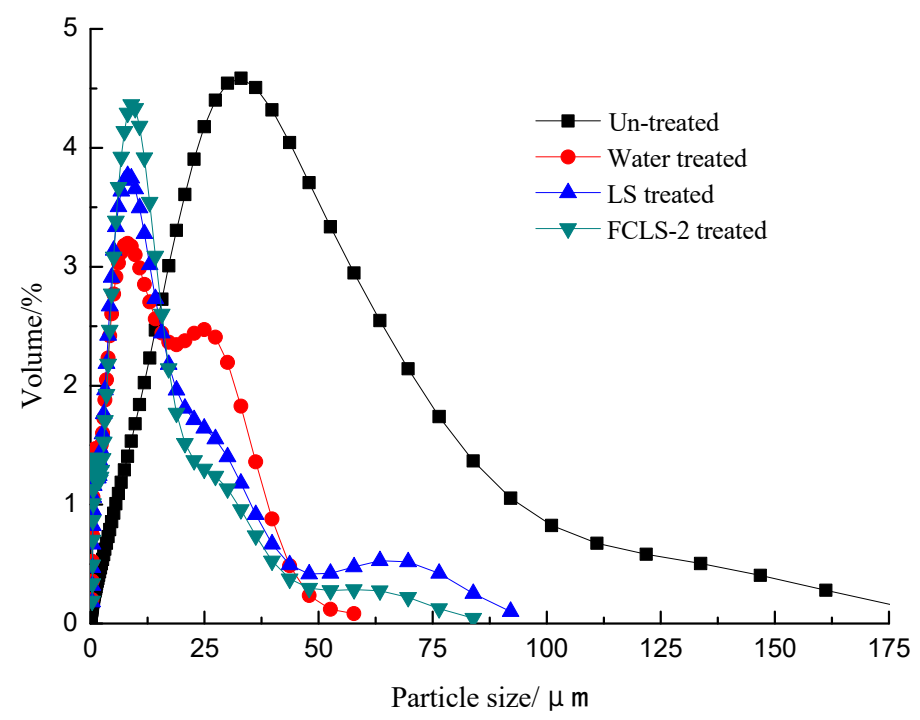

Figure 4. The distribution of clay particle size in different suspensions.

Table 3. Particle size of bentonite treated with different methods.

\begin{tabular}{ccc}
\hline Additives $\left(\mathbf{1 8 0}{ }^{\circ} \mathbf{C}\right)$ & Mean $/ \boldsymbol{\mu m}$ & Median $/ \boldsymbol{\mu m}$ \\
\hline Un-treated & 38 & 27 \\
Water treated & 8 & 5 \\
$0.3 \%$ LS & 8 & 6 \\
$0.3 \%$ FCLS-2 & 10 & 8 \\
\hline
\end{tabular}

\subsection{TGA}

The ratio of mass loss and temperature is a crucial factor to evaluate the inhibitor [11]. The temperature-dependent weight loss of different modified bentonite samples was measured using TGA technology as shown in Figure 5. The weight loss-temperature curves of FCLS-2 or LS-treated bentonites significantly differs from the control from $50{ }^{\circ} \mathrm{C}$ to $180^{\circ} \mathrm{C}$, particularly the former. The weight loss of tap water-treated bentonite was up to $3.2 \%$ from $50^{\circ} \mathrm{C}$ to $180^{\circ} \mathrm{C}$, while that of the $0.3 \%$ FCLS- 2 modified bentonite was down to $0.32 \%$. This obvious difference indicated that the FCLS- 2 can reduce the water amount of the sample except that the organic compounds will decompose above $180^{\circ} \mathrm{C}$. The result also revealed that FCLS-2-treated bentonites possess better thermal stability than LS-treated samples in the temperature above. 


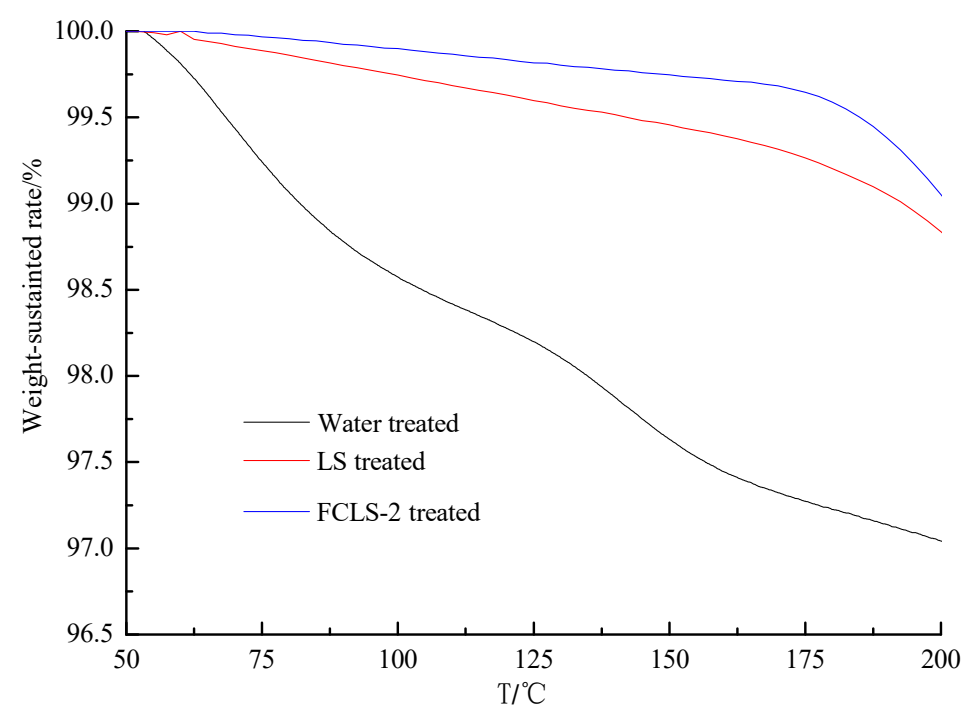

Figure 5. The thermogravimetric analysis (TGA) of different bentonite samples.

\subsection{SEM}

In order to further study the inhibition mechanism of FCLS-2, SEM was used to observe the morphology of bentonite with different modification. SEM images displayed that the particle size of $0.3 \%$ FCLS-2-immersed bentonite was much larger than tap water-treated bentonite after $24 \mathrm{~h}$ as shown in Figure 6. The result was consistent with their distribution of clay particle size determined by laser particle size analyzer. On the whole, the performance of FCLS-2 not only prevent bentonite from hydrating and swelling, but also pull and gather most of the hydrated and swollen clay [12,13].

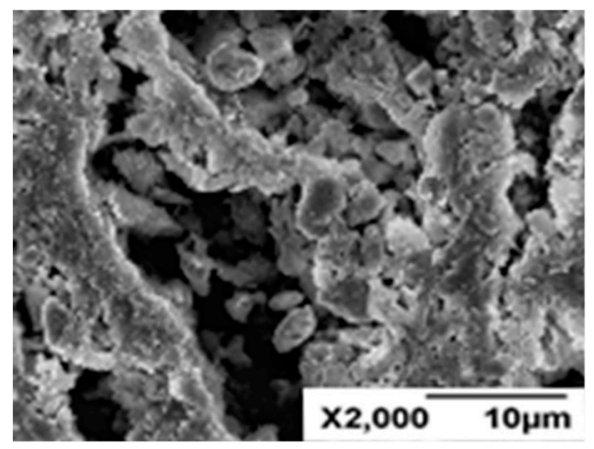

(A)

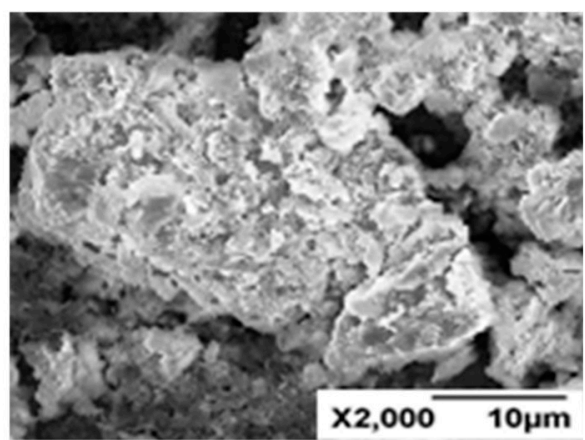

(B)

Figure 6. Scanning electron microscopy image (SEM) images of bentonites: (A) hydrated bentonite; (B) bentonite treated with FCLS-2.

\section{Materials and Methods}

\subsection{Materials and Reagents}

Sodium lignin sulfonate and ferric chloride were purchased from Xi'an Chemical Reagent Co. Ltd. (Xi'an, China). Bentonite, modified-starch, and PAM were all supplied from Yanchan Oilfield chemical company (Shaanxi, China).

\subsection{Preparation of FCLS}

Sodium lignin sulfonate (LS) and ferric chloride (FC) were dissolved in distilled water respectively with the concentration of $20 \%$. Then ferric chloride solution was added into the lignin sulfonate 
solution with different mass ratio dropwise under stirring at room temperature [14,15]. As shown in Figure 7, the final product, ferric chloride-lignin sulfonate (FCLS) solution was achieved after stirring for $1 \mathrm{~h}$ at $60 \mathrm{r} / \mathrm{min}$.
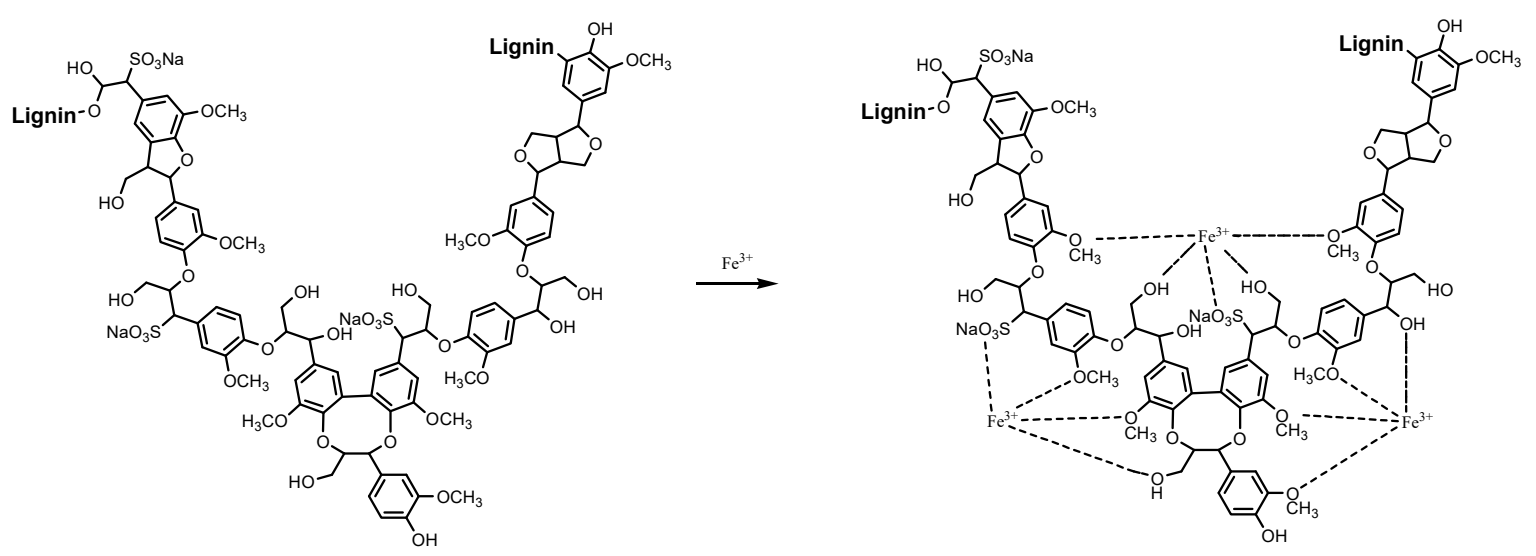

Figure 7. Synthetic principle of ferric chloride-lignin sulfonate inhibitor (FCLS).

\subsection{Evaluation of Inhibitive Ability}

The swelling behavior of bentonite in water was evaluated using a shale expansion instrument (NP-01, Haitongda, Co., Ltd., Chuangmeng, Qingdao, China), according to Chinese Petroleum and Natural Gas Industry Standards SY/T5971-1994 and SY/T6335-1997. Mud ball immersed experiment was conducted as follows: $10 \mathrm{~g}$ of bentonite and $10 \mathrm{~mL}$ of tap water were used to form a mud ball, and then it was placed in $80 \mathrm{~mL}$ of aqueous solutions or tap water for $24 \mathrm{~h}[16,17]$. The appearances of the mud balls were recorded and compared. The general error of the data is $\pm 0.2 \%$.

\subsection{Performance in Drilling Fluid}

The water based drilling fluid was prepared with a dosage of $4 \%$ bentonite $(\mathrm{m} / \mathrm{m})$, and the apparent viscosity (AV), plastic viscosity (PV), yield point (YP), API Filtration (FL), and friction coefficient (tg) was evaluated using a viscometer (ZNN-D6S, Haitongda, Co., Ltd., Qingdao, China) according to the reported methods [18-20]. The general error of the data is $\pm 1 \%$.

\subsection{Particle Distribution Measurement}

The bentonite was dispersed in a certain solution or tap water with a dosage of $4 \%$ ( $w / w)$, and was stirred for $24 \mathrm{~h}$. After inhibitor was added to the system, it was stirred for another $20 \mathrm{~min}$. Then the particles size distribution was measured by a laser particle size analyzer (LS-13320, Beckman Coulter, Inc., Brea, CA, USA) under the reported method [14,15]. The general error of the data is $\pm 2 \%$.

\subsection{TGA and SEM}

The bentonite samples were dispersed in tap water or system with inhibitor over $24 \mathrm{~h}$. Then, the bentonite was separated from the system and dried at $105{ }^{\circ} \mathrm{C}$ for TGA and SEM image. TGA experiment was conducted on a TGA/DSC thermal analysis instrument (1/1600, METTLER TOLEDO, Inc., Columbus, $\mathrm{OH}, \mathrm{USA}$ ) at a ramp of $20^{\circ} \mathrm{C} / \mathrm{min}$ from room temperature to $825{ }^{\circ} \mathrm{C}$ under nitrogen flow. The surface morphology of the bentonite samples was evaluated by a digital microscope imaging scanning electron microscope (model SU6600, serial No. HI-2102-0003) at a $40.0 \mathrm{kV}$ accelerating voltage on the basis of the reported method [14,15].

\section{Conclusions}

In this work, ferric chloride-lignin sulfonate (FCLS) was synthesized with ferric chloride and lignin sulfonate and their performance as shale inhibitor in water-based drilling fluid was systematically 
investigated. The result demonstrated that FCLS-2 displays effectively inhibitive ability against the hydrating and swelling processes of clay. The swelling rate of $0.3 \%$ FCLS- 2 treated clay decreased from $69.0 \%$ to $41.9 \%$. In addition, the mud ball added FCLS- 2 was more stable, and its swelling degree was much lower compared with control. Furthermore, the inhibition mechanism of FCLS-2 to shales was explored using SEM combined with TGA and particle size analyzer. The probable mechanism is that FCLS-2 not only prevents bentonite from hydrating and swelling via hydrogen bonding, ion exchange, and anchoring effect, but also pulls and gathers most of the hydrated and swollen clay.

Author Contributions: Conceptualization, G.C. and J.Z.; methodology, X.G. and J.Z.; validation, W.D. (Wenguang Duan) and R.Z.; formal analysis, L.G.; investigation, R.Z.; resources, W.D. (Weichao Du); data curation, W.H.; writing-original draft preparation, R.Z.; writing-review and editing, G.C.; supervision, J.Z.; project administration, G.C.; funding acquisition, J.Z.

Funding: The work was supported financially by National Science and Technology Major Project (No. 2017ZX05069004), Shaanxi Provincial Key Research and Development Program (No. 2019ZDLGY06-03) and Scientific Research Program Funded by Shaanxi Provincial Education Department (No. 18JS089).

Conflicts of Interest: The authors declare that they have no competing interests.

\section{References}

1. Chen, G.; Yan, J.; Li, L.L.; Zhang, J.; Gu, X.F.; Song, H. Preparation and performance of amine-tartaric salt as potential clay swelling inhibitor. Appl. Clay Sci. 2017, 138, 12-16. [CrossRef]

2. Muhammad, S.K.; Abdullah, S.S.; Usamah, A.A.; Ibnelwaleed, A.H. Review on polymer flooding: Rheology, adsorption, stability, and field applications of various polymer systems. Polymer Rev. 2015, 55, 491-530.

3. Gu, X.F.; Zhang, J.J.; Zhang, J.; Chen, G.; Ma, C.; Zhang, Z.F. Stabilization of montmorillonite by ammoniated lignosulfonates and its use in water-based drilling fluid. Sci. Adv. Mater. 2017, 9, 928-933. [CrossRef]

4. Chen, G.; Zhang, J.; Yang, N.W.; Ma, Y.F. The evaluation of sodium hydroxymethyl lignosulfonate as an ecofriendly drilling fluid additive. Pet. Sci. Technol. 2014, 32, 1816-1823. [CrossRef]

5. Zhang, J.; Chen, G.; Yang, N.W.; Wang, Y.G. Preparation of nitration-oxidation lignosulfonate as an ecofriendly drilling fluid additive. Pet. Sci. Technol. 2014, 32, 1661-1668. [CrossRef]

6. Ahmed, H.M.; Kamal, M.S.; Al-Harthi, M.A. Polymeric and low molecular weight shale inhibitors: A review. Fuel 2019, 251, 187-217. [CrossRef]

7. Chen, G.; Zhang, J.; Cheng, C.; Sun, Y.; Hu, Q.; Qu, C.T.; Dong, S.B. Synergistic effect of surfactant and alkali on the treatment of oil sludge. J. Pet. Sci. Eng. 2019, 183, 106420. [CrossRef]

8. Wu, J.K.; Xu, H.Y.; Xie, Y.N.; Zhou, X.Y. A research and application of strong inhibition drilling fluid. Appl. Mech. Mater. 2014, 675, 1481-1484. [CrossRef]

9. Yin, X.C.; Zhang, L.M.; Li, Z.M. Studies on new ampholytic cellulose derivative as clay-hydration inhibitor in oil field drilling fluid. J. Appl. Polym. Sci. 1998, 70, 921-926. [CrossRef]

10. Han, Y.Z.; Song, Z.Q.; Huang, W.A.; Cao, J. Shale inhibitive properties of polyether diamine in water-based drilling fluid. J. Pet. Sci. Technol. 2011, 78, 510-515.

11. Xi, Y.F.; Ding, Z. Structure of organoclays-an X-ray diffraction and thermogravimetric analysis study. J. Colloid Interface Sci. 2004, 277, 116-120. [CrossRef] [PubMed]

12. Wang, Y.L.; Yan, Q.B.; Guo, G.; Guo, Z.; Zhang, J.; Chen, G.; Deng, Q. Investigation of oleate graft copolyammonium as a clay swelling inhibitor for shale oil/gas exploration. Petrol. Chem. 2018, 58, 255-259. [CrossRef]

13. Ye, Z.Q.; Ye, Z.B.; Huang, L.; Bai, Y.; Li, L.L.; Zhang, J.; Qu, C.T.; Chen, G. Preparation and application of a new cross-linked polyammonium as shale inhibitor. J. Appl. Biomater. Funct. Mater. 2018, 16, 119-124.

14. Zhang, J.; Hu, W.M.; Zhang, L.; Li, T.H.; Cai, D.; Chen, G. Investigation of ammonium-lauric salt as shale swelling inhibitor and a mechanism study. Adsorpt. Sci. Technol. 2019, 37, 49-60. [CrossRef]

15. Gao, B.Y.; Sun, X.; Yue, Q.Y.; Zhang, D.H. The structure of epichlorohydrin-dimethylamine polymer flocculants with different modifying agents and their properties for decoloration of wastewater. Acta Sci. Circumstantiae 2006, 26, 1977-1982.

16. Zhang, L.; Li, T.H.; Huang, L.; Qin, Y.Z.; Bin, Y.Z.; Yan, X.; Li, L.L.; Deng, Q. Preparation and application of melamine cross-linked poly ammonium as shale inhibitor. Chem. Central J. 2018, 12, 44. [CrossRef] [PubMed] 
17. Cai, D.; Zhang, J.; Chen, G. Study on the effect of carboxy-amines small molecule shale inhibitor. Oilfield Chem. 2014, 31, 5 .

18. Zhang, J.; Zhang, Q.; Chen, G. Mechanism study and application of modified polysaccharides as drilling fluid additive with high inhabitability. Technol. Dev. Chem. Ind. 2013, 42, 1-5.

19. Chen, G.; Gao, L.; Dong, K.; Sun, Y.; Hu, W.M. A green shale inhibitor developed from lignin sulfonate and a mechanism study. J. Biobased. Mater. Bio. 2019, 13, 778-783.

20. Petroleum, A.P.I. Petroleum and Natural Gas Industries for Field Testing of Drilling Fluids. Available online: https://www.iso.org/standard/41939.html (accessed on 15 June 2001).

Sample Availability: Samples of the Ferric Chloride-Lignin Sulfonates are available from the authors.

(C) 2019 by the authors. Licensee MDPI, Basel, Switzerland. This article is an open access article distributed under the terms and conditions of the Creative Commons Attribution (CC BY) license (http://creativecommons.org/licenses/by/4.0/). 